\title{
Attenuation From Shoes and Pads in Equine Nuclear Scintigraphy, With Relevance to Solar Views
}

\section{OPEN ACCESS}

Edited by:

Fintan John McEvoy,

University of Copenhagen, Denmark

Reviewed by:

Andrew Bathe,

Rossdales Equine Hospital,

United Kingdom

Seamus Edward Hoey,

University College Dublin, Ireland

*Correspondence:

Lea Walker

leawalkerdvm@yahoo.com

tPresent address:

Bianca Drumond,

VetAgro Sup - Campus Vétérinaire de

Lyon, Marcy-l'Etoile, France

Lea Walker,

Equine Sports Medicine and Imaging,

Waddell, AZ, United States

Specialty section: This article was submitted to

Veterinary Imaging,

a section of the journa

Frontiers in Veterinary Science

Received: 02 December 2019

Accepted: 19 August 2020

Published: 24 September 2020

Citation:

Walker L, Martinelli MJ, Rantanen N, Drumond B and Trostle S (2020)

Attenuation From Shoes and Pads in

Equine Nuclear Scintigraphy, With

Relevance to Solar Views.

Front. Vet. Sci. 7:516718.

doi: 10.3389/fvets.2020.516718

\author{
Lea Walker ${ }^{1 * t}$, Mark J. Martinelli ${ }^{1}$, Norman Rantanen ${ }^{1}$, Bianca Drumond ${ }^{1 \dagger}$ and \\ Steven Trostle ${ }^{2}$ \\ ${ }^{1}$ California Equine Orthopedics, San Marcos, CA, United States, ${ }^{2}$ Blue Ridge Equine Clinic, Earlysville, VA, United States
}

Nuclear scintigraphy can be beneficial in the investigation of equine lameness and poor performance. Images obtained through the sole allow for better identification of a region of increased radionuclide uptake in the foot. The presence of shoes or pads may affect these images. To determine the degree of radioactive attenuation different types of shoes and pads of different thickness and materials were evaluated by placing the material directly on the gamma camera detector acquiring flood images from a point source. The study compared five different types of shoes from 3 different shoeing materials, steel, aluminum, and polyurethane. This study also assessed 8 different types of pads that were selected based on the subjective densities and variable rigidity of the materials. All types of shoes/pads evaluated caused some degree of attenuation $(P<0.05)$. Steel shoes of all types cause the most attenuation (54\%), followed by aluminum shoes $(22 \%)$, and the shoes or pads composed primarily of a plastic polymer cause the least amount of attenuation (15\%). The results of the study found that pads or shoes cause significant $(p<0.05)$ attenuation of gamma radiation. Composition, thickness and density characterized mass absorption coefficient, of the material affect the amount of gamma radiation attenuation. Because of the attenuation of gamma radiation, we recommend shoes and pads be removed prior to nuclear scintigraphic examination of the front foot if a solar image is desired, as this attenuation may affect image quality.

Keywords: equine, scintigraphy, attenuation, shoe, Pad, solar view

\section{INTRODUCTION}

Nuclear scintigraphy of the equine foot is an established imaging modality in the investigation of equine lameness and poor performance (1). Increased radiopharmaceutical uptake (IRU) has been described by researchers and clinicians in association with abnormalities of the navicular bone (1-5) collateral ligaments of the distal interphalangeal joint $(2,3,6)$, deep digital flexor tendon $(1,2)$, distal phalanx $(2,7,8)$ and ungular cartilages $(9-11)$ and used to evaluate laminar perfusion (12). Nuclear scintigraphy of the equine musculoskeletal system has been considered to be very sensitive, but not specific for injuries in lame or poorly performing horses $(1,2)$. Although MRI, and to a lesser degree $\mathrm{CT}$, are the predominant techniques for advanced imaging of the foot, nuclear scintigraphy can provide additional clinically useful information. The value of nuclear scintigraphy has been documented for several anatomic sites within the foot $(1,3)$. The agreement between both increased radiopharmaceutical uptake and relevance categorization with the presence of foot pain is substantial (13), and a lack of radiopharmaceutical uptake does not preclude injury and can still provide potentially important information in the foot (7). 
For assessment of uptake of the structures in the foot, lateral/medial, dorsal/palmar and solar scintigraphic views are necessary $(3,9,14)$. Different types of shoes or shoe/pad combinations may reduce the ability of the practitioner to clearly identify the different structures, making accurate diagnosis more difficult, especially on the solar views. There is limited information correlating types of shoes and pads with the amount of attenuation of the radiation. It has been recommended in some circumstances, particularly with eggbar and heartbar shoes that may effectively act as a mask or normal open shoes that shield parts of the bones of the foot, to remove the shoes to obtain an adequate solar image (15).

The purpose of this study was to quantify the percent attenuation of gamma radiation by different types and materials of shoes and pads commonly worn by horses undergoing nuclear scintigraphic imaging. It is hypothesized that different types and materials of shoes and pads will cause attenuation of the solar view. Attenuation of gamma radiation effectively increases the noise in the data received by the detector, even if the attenuation is uniform across the solar view. Therefore, we propose in a clinical setting that in the presence of a shoe or pad some information will be inadvertently masked or lost, which may impact the ability of the clinician to accurately interpret a solar image.

\section{MATERIALS AND METHODS}

\section{Imaging, Instrument Calibration, and Uniformity}

Images were acquired on a NuCam gamma camera (Anger Type) mounted on an EqView stand both produced by Diagnostic Services Inc. (Middlesex, NJ) and interfaced to a Mirage Imaging Computer produced by Segami Corp (Columbia, MD). The camera includes a $0.95-\mathrm{cm}$ thick $\mathrm{NaI}$ (sodium iodide) crystal optically interfaced to 55 photomultiplier tubes. Energy level and $\mathrm{X}-\mathrm{Y}$ position of gamma events are determined at the detector. The Mirage computer digitizes the signal, applies isotope selection, energy window selection and applies energy, linearity, and uniformity corrections. Images were acquired in a 256 by 256 matrix on a $51-\mathrm{cm}$ by $37-\mathrm{cm}$ field of view. Flood images were generated using a calibration dose (37 Mbq/1.0 mCi) of radioactive technetium-99 $\left({ }^{99 \mathrm{~m}} \mathrm{Tc}\right)$ as sodium pertechnetate, placed in small tube and located several meters away from the camera. Radioactive ${ }^{99 \mathrm{~m}} \mathrm{Tc}$ emits a $140 \mathrm{keV}$ gamma ray with a half-life of $6.0 \mathrm{~h}$. NEMA (National Electrical Manufacturers Association) internal uniformity tests were performed and passed for use of the instrument. The NuCam gamma camera assembly includes a lead collimator to enhance image focus and resolution. The collimator was removed to ensure that a uniform radiation background is obtained and to reduce the data acquisition times.

\section{Control and Attenuation Data Acquisition}

Internal calibration and uniformity testing of the instrument was completed. Randomized multiple sets of shoes or pads were placed directly on the surface of the camera and flood images of the field were acquired to total 3,000,000 counts. A total of four "sets" of shoe and/or pad images were obtained, each set consisting of a total of as many as four shoes or pads per camera view. Data was acquired for each set three times to reduce imaging error. The three acquisitions were compared to each other to confirm reproducibility and field uniformity. One set was acquired four times. The fourth image of that set was acquired for a total of 12,000,000 counts to accurately trace the margins of the pads in that image. Additionally, the higher counts also improve the statistical uncertainties for the pads. The acquired images are in Appendix 1.

A summary of the materials used in each of the four sets of data is summarized in Table 1. In each image acquired, measurements of identical areas (ROIs) in three unobstructed "control" locations on the field (measured in points through the instrument's Mirage software, and points are analogous to pixels on a computer screen) were taken to determine the number of counts in a given area (counts per point). The "control" incident radiation detected as counts/point is designated $I_{0}$.

Additionally, for each data set, the outline of each shoe or pad was traced creating a ROI. The area and the counts for each ROI were generated ( $I$, as counts per point). Due to the variable thickness of the pads and some shoes, two types of ROI (I) measurements were performed. Small, uniform circular areas in multiple locations were measured and also, the entirety of the pad or shoe was traced.

The average of these control measurements within and across acquisitions for a set was used to evaluate the degree of attenuation of a given shoe or pad ROI. The counts per point for each shoe or pad ROI (I) in each data set was compared to the average control counts per point in each data set and percent attenuation was generated (16).

$$
\text { Attenuation }=A=\left[1-I(\text { shoe } / \text { pad }) / I_{0}(\text { control })\right] * 100
$$

These data and results of the attenuation calculations are presented in the Results (section Results). In the data sets, estimates of $I_{0}$ use small circular ROIs traced over portions of the image where no obstructing materials exist. For example, in Set 1 there are five control ROIs used over a total of three acquisitions of data. In the first acquisition, there are three control ROIs located (a) at the upper left, (b) at the image center, and (c) at the bottom center. Acquisitions 2 and 3 have a control ROI located at the center of the images identical in size and location to the central ROI in acquisition 1 . These 5 ROIs were used to confirm the spatial field uniformity across the camera face in a single

TABLE 1 | Sets of Shoes/Pads Imaged Concurrently.

\begin{tabular}{llll}
\hline Set 1 & Set $\mathbf{2}$ & Set $\mathbf{3}$ & Set $\mathbf{4}$ \\
\hline $\begin{array}{l}\text { Standard steel } \\
\text { shoe }\end{array}$ & $\begin{array}{l}\text { Green impact } \\
\text { pad }\end{array}$ & $\begin{array}{l}\text { Aluminum spider } \\
\text { plate }\end{array}$ & $\begin{array}{l}\text { Small aluminum } \\
\text { wedge shoe }\end{array}$ \\
$\begin{array}{l}\text { Steel heartbar } \\
\text { shoe }\end{array}$ & $\begin{array}{l}\text { Black impact } \\
\text { pad }\end{array}$ & Black spider plate & $\begin{array}{l}\text { Large aluminum } \\
\text { standard shoe }\end{array}$ \\
$\begin{array}{l}\text { Steel eggbar } \\
\text { shoe }\end{array}$ & $\begin{array}{l}\text { Leather impact } \\
\text { pad }\end{array}$ & Waffle impact pad & Plastic shoe \\
$\begin{array}{l}\text { Small aluminum } \\
\text { wedge shoe }\end{array}$ & $\begin{array}{l}\text { Clear wedge } \\
\text { impact pad }\end{array}$ & Plastic shoe & Green rim pad
\end{tabular}


acquisition and the temporal field uniformity at a single spot in the center of each image obtained in three successive acquisitions.

\section{Effect of Shoes and Pads}

The study compared five different types of shoes (standard aluminum (2 sizes), standard steel, steel eggbar, steel heartbar, and plastic (Epona, Epona Shoe, Inc., Creston, CA 93432 USA) made from 3 different shoeing materials. This study also assessed several different types of pads. A black plastic impact pad, a green plastic impact pad, a clear plastic wedge pad with frog support, a black plastic spider plate, a green rim pad, an aluminum spider plate, a leather impact pad and a "waffle-pad" were all evaluated. The different plastic materials were chosen based on the subjective densities and variable rigidity of the materials. The clear plastic material was hardest and most rigid of them, whereas the waffle-pad was the softest and most pliable. The green plastic impact pad and rim pad were slightly more rigid than the waffle pad and the black plastic materials (impact pad and spider plate) were slightly less rigid than the clear plastic pad. The thicknesses of the shoes and pads are listed in Table 2.

\section{Statistical Analysis of Background Measurements, Shoe and Pad Attenuation Data}

Statistical analysis of background ROIs and of the shoe and pad ROI data was performed using ANOVA calculations through a web-based interface at (URL: http://statpages.info/anova1sm. html, retrieved 10/15/2018), using total gamma ray counts, count rate means, and standard errors of the means (the uncertainties in the count rates alone). The standard errors of the mean decrease with larger counts and larger ROIs (number of points).

TABLE 2 | Shoe and Pad Materials and Thicknesses.

\begin{tabular}{|c|c|c|}
\hline & Material & Thickness \\
\hline \multicolumn{3}{|l|}{ Shoes } \\
\hline Standard & Steel & $7.5 \mathrm{~mm}$ \\
\hline Standard & Aluminum & Heel- 13.5 mm \\
\hline Heartbar & Steel & $9.0 \mathrm{~mm}$ \\
\hline Eggbar & Steel & $8.0 \mathrm{~mm}$ \\
\hline Small wedge & Aluminum & Heel- $13.9 \mathrm{~mm}$ \\
\hline Plastic & Polyurethane & $14.5 \mathrm{~mm}$ \\
\hline \multicolumn{3}{|l|}{ Pads } \\
\hline Impact pad & $\begin{array}{l}\text { Soft green plastic } \\
\text { wedge }\end{array}$ & Heel- $7.3 \mathrm{~mm}$ \\
\hline Impact pad & Hard black plastic & $5.0 \mathrm{~mm}$ \\
\hline Impact pad & Leather & $5.3 \mathrm{~mm}$ \\
\hline \multirow[t]{2}{*}{ Impact pad } & Hard clear wedge & Heel- $11.3 \mathrm{~mm} \quad$ Toe- $1.8 \mathrm{~mm}$ \\
\hline & with frog support & Frog- $12.0 \mathrm{~mm}$ Frog center- $4.3 \mathrm{~mm}$ \\
\hline Impact pad & $\begin{array}{l}\text { Soft clear "waffle" } \\
\text { plastic }\end{array}$ & $5.3 \mathrm{~mm}$ \\
\hline Impact pad & Green rim & $5.0 \mathrm{~mm}$ \\
\hline Spider plate & Aluminum & $3.0 \mathrm{~mm}$ \\
\hline Spider plate & Black plastic & $5.5 \mathrm{~mm}$ \\
\hline
\end{tabular}

Descriptive statistical means and standard deviations of the means were calculated and summarize the percentage of attenuation by different shoes and pads. Comparison of gamma radiation attenuation measurements was performed using repeated ANOVA calculations. For all analysis, $p<0.05$ were considered statistically significant.

\section{RESULTS}

\section{Summary Data for Background Measurements}

Data for unobstructed regions of interest $\left(I_{0}\right)$ can be found in Table 3. Control measurements for each data set $\left(I_{0}\right)$ confirm the uniformity of the field and normalize for radiopharmaceutical decay during the procedure. Counts and points in the table are raw data taken from information printed on the generated images using the Mirage Imaging software.

\section{Statistical Analysis of Background Measurements}

An ANOVA was used to calculate the means and standard deviations of ROIs 0,1 , and 2 from acquisition 1 of Set 1 (Table 3). The corresponding ANOVA calculation returns a $p$ value of $p=0.80$, indicating the amount of variation in the count rates for these three ROIs would be expected $\sim 80 \%$ of the time if the samples are taken from the same underlying distribution. Secondly, if each field is uniform between two different acquisitions made for a given set of data, then it will not matter if the control ROI is extracted from one acquisition and the shoe/pad ROI is extracted from a subsequent or prior acquisition. ANOVA was used to compare the means and standard deviations of ROI 0 from acquisitions 1, 2, and 3 of the Set 1 data, also in Table 3. This ANOVA calculation returns a $p$-value of $p=0.86$, indicating we can expect to see this much variation between means $\sim 86 \%$ of the time. The background measurements and analysis for each set $\left(I_{0}\right)$ in Table 3 confirm the uniformity of the field and from one acquisition to the next. This process also normalizes for radiopharmaceutical decay during the procedure since it is concurrent with the ROI measurements of the shoes and pads.

The measured background, used to calculate values of $I_{0}$ is statistically uniform, both within a single acquisition image and from one acquisition to the next.

\section{Summary Data for Shoes and Pads}

Data for shoes/pads and calculated attenuation (1-I/Io)100\% using equation 1 is presented in Tables 4, 5 and a summary of the observed attenuation data is presented graphically in Figure 1. Counts and points in the tables are raw data taken from information printed on the generated images using the Mirage Imaging software. The corresponding average $I_{0}$ for each set is found in Table 3. An expression for the uncertainties associated with the measurements was derived using calculus of variations, so that

$$
\delta(A)=100 \alpha \frac{C_{M}^{1 / 2}}{C_{B}}\left(1+\frac{C_{M}^{1 / 2}}{C_{B}^{1 / 2}}\right)
$$


TABLE 3 | Counts per point in unobstructed regions of interest in each of 4 image sets.

\begin{tabular}{|c|c|c|c|c|c|}
\hline Set \# & Location on image & Image in set & Counts & Points (pixels) & Counts/point \\
\hline Set 1 & $\mathrm{ROI} 0$ & Image 1 & 10,964 & 208 & 52.71 \\
\hline Set 1 & $\mathrm{ROI} 1$ & Image 1 & 11,011 & 209 & 52.68 \\
\hline Set 1 & $\mathrm{ROI} 2$ & Image 1 & 11,097 & 209 & 53.10 \\
\hline Set 1 & $\mathrm{ROI} 0$ & Image 2 & 11,023 & 208 & 53.00 \\
\hline Set 1 & $\mathrm{ROI} 0$ & Image 3 & 10,946 & 208 & 52.63 \\
\hline Set 1 & Average $\mathrm{I}_{\circ}$ & & & & 52.82 \\
\hline Set 2 & $\mathrm{ROI} 0$ & Image 1 & 10,502 & 208 & 50.49 \\
\hline Set 2 & $\mathrm{ROI} 1$ & Image 1 & 10,720 & 210 & 51.05 \\
\hline Set 2 & $\mathrm{ROI} 2$ & Image 1 & 10,857 & 209 & 51.95 \\
\hline Set 2 & $\mathrm{ROI} 0$ & Image 2 & 10,579 & 208 & 50.86 \\
\hline Set 2 & $\mathrm{ROI} O$ & Image 3 & 10,548 & 208 & 50.71 \\
\hline Set 2 & Average $\mathrm{I}_{\circ}$ & & & & 51.01 \\
\hline Set $2^{*}$ & $\mathrm{ROI} 0$ & Image $4^{*}$ & 42,268 & 208 & 203.21 \\
\hline Set 2 & $I_{0}$ & Image 4 & & & 203.21 \\
\hline Set 3 & ROI 0 & Image 1 & 10,508 & 208 & 50.52 \\
\hline Set 3 & ROI 1 & Image 1 & 10,626 & 209 & 50.84 \\
\hline Set 3 & ROI 2 & Image 1 & 10,492 & 210 & 49.96 \\
\hline Set 3 & $\mathrm{ROI} 0$ & Image 2 & 10,476 & 208 & 50.37 \\
\hline Set 3 & ROI 0 & Image 3 & 10,465 & 208 & 50.31 \\
\hline Set 3 & Average $\mathrm{I}_{\circ}$ & & & & 50.40 \\
\hline Set 4 & ROI 0 & Image 1 & 10,648 & 208 & 51.19 \\
\hline Set 4 & ROI 1 & Image 1 & 10,813 & 208 & 51.99 \\
\hline Set 4 & ROI 2 & Image 1 & 10,747 & 209 & 51.42 \\
\hline Set 4 & $\mathrm{ROI} 0$ & Image 2 & 10,615 & 208 & 51.03 \\
\hline Set 4 & ROI 0 & Image 3 & 10,472 & 208 & 50.35 \\
\hline Set 4 & Average $\mathrm{I}_{\mathrm{o}}$ & & & & 51.20 \\
\hline
\end{tabular}

Bolded values are the averages from each set.

where $\alpha$ is the ratio of background counts to counts when obstructing materials are present, and $C_{M}$ and $C_{B}$ are the total counts in a region of interest that contains materials $\left(C_{M}\right)$ or only contains background counts $\left(C_{B}\right)$.

The uncertainties reported in Tables 4, 5 depend on the total number of counts accumulated in the regions of interest. The uncertainty in the number of counts follows from the fact that gamma ray detection follows a Poisson distribution (16), so that the corresponding uncertainty in counts for a ROI is the square root of the total counts. All statistical analyses were performed by Robert $\mathrm{B}$. Walker, $\mathrm{PhD}$ and a complete derivation of this expression is available by request to the first author.

\section{Statistical Analysis of Shoe and Pad Attenuation Data}

ANOVA confirms that although the center and rim attenuation measurements of the aluminum spider plate have slightly different attenuations (2.8 vs. $5.7 \%)$, they are not statistically different $(p=0.17$ ) from each other. For the Large Aluminum Standard shoe, the heel and toe attenuations are 27.8 and $20.1 \%$, respectively, corresponding to maximum thickness measurements of 13.5 and $9.5 \mathrm{~mm}$ (although average effective thicknesses are smaller, due to irregularities in the shoe). ANOVA calculations demonstrate that these attenuation differences are statistically significant, $p=0.0008$, in part because the attenuations are higher as a whole. The overall attenuation for the Large Aluminum Shoe was $22.4 \%$, closer in value to the toe measurement since this shoe has a thicker heel. An ANOVA calculation comparing the whole shoe, and the toe and rim, shows that the count rates for the heel are significantly different than for the overall shoe or the toe ( $p=0.0022$ and 0.0044$)$, but the shoe and toe count rates are not significantly different (at $p=0.31$ ).

Differences in count rates can occur within a single pad. The clear pad shows definite structure in the acquired image data, with the toe producing the least attenuation $(2.99 \%)$ and the frog heel portion producing the most $(18.0 \%)$, with associated maximum thickness measurements of 1.8 and $12.0 \mathrm{~mm}$. ANOVA calculations show the spot ROI count rates to be statistically different at $p=0.029$.

When comparing classes of shoes (Steel vs. Aluminum vs. Plastic), using the combined counts and ROIs for the three steel shoes, the two aluminum shoes, and the plastic shoe, all three sets of attenuations are statistically significant (with $p<0.0001$ ).

\section{DISCUSSION}

All shoes regardless of type or material caused some degree of attenuation of gamma radiation. As a group, shoes caused noticeably more gamma radiation attenuation than the pads. Regardless of the shoe type, shoes made of steel (standard, 
TABLE 4 | Data for Shoes (I) and Attenuation Calculation.

\begin{tabular}{|c|c|c|c|c|c|c|}
\hline $\begin{array}{l}\text { Set \#/ } \\
\text { image\#/ } \\
\text { location }\end{array}$ & ROI type & $\begin{array}{l}\text { Material description } \\
\text { thickness }(\mathrm{mm})\end{array}$ & Counts & $\begin{array}{l}\text { Points } \\
\text { (pixels) }\end{array}$ & $\begin{array}{l}\text { Counts/point } \\
\text { (I) }\end{array}$ & $\begin{array}{c}\left(1-I / I_{0}\right) 100 \% \text { percent } \\
\text { attenuation } \\
\pm \text { uncertainty calc. } \\
\text { from equation } 2\end{array}$ \\
\hline \multicolumn{7}{|l|}{ Shoes } \\
\hline Set 1/image 3/ROI 0 & Full trace & $\begin{array}{l}\text { Steel- heartbar } \\
9.0 \mathrm{~mm}\end{array}$ & 41,065 & 1,695 & 24.23 & $54.13 \pm 0.42^{a}$ \\
\hline Set 1/image $3 / \mathrm{ROI} 1$ & Full trace & $\begin{array}{l}\text { Steel - standard } \\
7.5 \mathrm{~mm}\end{array}$ & 26,239 & 1,057 & 24.82 & $53.00 \pm 0.49^{a}$ \\
\hline Set 1/image 3/ROI 2 & Full trace & $\begin{array}{l}\text { Steel- eggbar } \\
3.0 \mathrm{~mm}\end{array}$ & 33,987 & 1,417 & 23.99 & $54.59 \pm 0.44^{a}$ \\
\hline Set 1/image 3/ROI 3 & Full trace & $\begin{array}{l}\text { Aluminum- small } \\
8.4-13.9 \mathrm{~mm}\end{array}$ & 61,562 & 1,471 & 41.85 & $20.77 \pm 0.66^{b}$ \\
\hline Set 4/image 3/ROI 3 & Full trace & $\begin{array}{l}\text { Aluminum- small } \\
8.4-13.9 \mathrm{~mm}\end{array}$ & 55,639 & 1,384 & 40.20 & $21.48 \pm 0.67^{b}$ \\
\hline Set $4 /$ image $3 / \mathrm{ROI} 1 \mathrm{a}^{\beta}$ & Spot area & $\begin{array}{l}\text { Aluminum small heel } \\
(13.9 \mathrm{~mm})\end{array}$ & 1,941 & 57 & 34.05 & $33.49 \pm 1.80 b$ \\
\hline Set $4 /$ image $3 / \mathrm{ROI} 3 \mathrm{a}^{\beta}$ & Spot area & $\begin{array}{l}\text { Aluminum small toe } \\
\text { (8.4 mm) }\end{array}$ & 2195 & 54 & 40.65 & $20.60 \pm 2.04^{b}$ \\
\hline Set 4/image 3/ROI 1 & Full trace & $\begin{array}{l}\text { Aluminum large } \\
9.5-13.5 \mathrm{~mm}\end{array}$ & 52,515 & 1,322 & 39.72 & $22.41 \pm 0.67^{b}$ \\
\hline Set $4 /$ image $3 / \mathrm{ROI} \mathrm{Oa}^{\beta}$ & Spot area & $\begin{array}{l}\text { Aluminum large heel } \\
(13.5 \mathrm{~mm})\end{array}$ & 2,070 & 56 & 36.96 & $27.80 \pm 1.90^{b}$ \\
\hline Set $4 /$ Image $3 / \mathrm{ROI} 2 \mathrm{a}^{\beta}$ & Spot area & $\begin{array}{l}\text { Aluminum large toe } \\
(9.5 \mathrm{~mm})\end{array}$ & 2,292 & 56 & 40.93 & $20.06 \pm 2.02^{b}$ \\
\hline Set 4/image 3/ROI 0 & Full trace & $\begin{array}{l}\text { Plastic } \\
14.5 \mathrm{~mm}\end{array}$ & 114,739 & 2,629 & 43.64 & $14.75 \pm 0.62^{c}$ \\
\hline Set 3/image 3/ROI 0 & Full trace & $\begin{array}{l}\text { Plastic } \\
14.5 \mathrm{~mm}\end{array}$ & 112,725 & 2,606 & 43.26 & $14.17 \pm 0.63^{c}$ \\
\hline Set 3/image 3/ROI 7 & Spot area & $\begin{array}{l}\text { Plastic center } \\
14.5 \mathrm{~mm}\end{array}$ & 3,551 & 82 & 43.30 & $14.08 \pm 1.82^{\mathrm{c}}$ \\
\hline
\end{tabular}

${ }^{\beta}$ Where a second copy of an image was used for additional ROls, a suffix designation "a" was added to indicate those ROls.

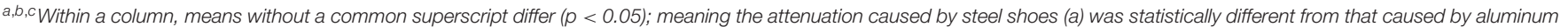
shoes (b) and both are statistically different from that caused by Plastic shoe (c).

eggbar and heart bar) provided the greatest average attenuation (53.9\%), followed by aluminum shoes $(21.59 \%)$ and then plastic shoes $(14.75 \%)$. All pads caused some degree of attenuation of gamma radiation. All the pads had $<10 \%$ average attenuation. The thickness of the pad and shoe material is the most important factor, aside from the material, in the amount of radiation that was attenuated, as evidenced the variable thickness of the clear plastic impact pad causing more attenuation at the thickest parts of the pad. But pads in general are thinner than shoes, typically cover the entire solar view, and so yield small overall attenuations. This is graphically represented in Figure $\mathbf{1}$.

At our $\gamma$-ray energy of $140 \mathrm{keV}$, the mass absorption coefficient is the sum of the partial mass absorption coefficients from the photoelectric and Compton scattering effects. As this physics has been well-described, tables of mass absorption coefficients in $\mathrm{cm}^{2} / \mathrm{g}$ are readily accessible in the literature (17). The mass absorption coefficient of Compton scattering decreases with $Z$ number while the photoelectric effect increases with $Z$ number. The elements of interest are iron- $Z=26$ (steel), aluminum- $Z=13$ and carbon- $Z=6$ (polymer such as polyurethane) since these are the elements that are of the highest concentration in the materials studied here. The total $\mu$ (mass absorption) coefficients, including both photoelectric and Compton scattering effects at near $140 \mathrm{keV}$ for iron, aluminum and carbon are $0.184(0.1240+0.0595), 0.134(0.1288+0.0052)$, and $0.134(0.1336+0.0003) \mathrm{cm}^{2} / \mathrm{g}$, respectively, where the numbers in parentheses are Compton scattering + photoelectric effect for each element.

To demonstrate the relationship of thickness to attenuation, Table 6 shows how predicted attenuations vary with thickness for each of the three major shoe materials in this study. The three parameters that define the predicted attenuation are the mass absorption coefficient, density, and thickness of the attenuating material. The first parameter depends on the element and the gamma ray photon energy previously discussed. For the second parameter we used common densities for steel and elemental aluminum but polymers may have a variety of densities depending on the manufacturing process (the error in the average density for Epona polyurethane could be different from the polymer in the table). A mid-range density of 1.2 $\mathrm{g} / \mathrm{cm}^{3}$ for polyurethane is used here. The third parameter is thickness but may not be as simple as a single measurement. 
TABLE 5 | Data for Pads/Plates (I) and Attenuation Calculation.

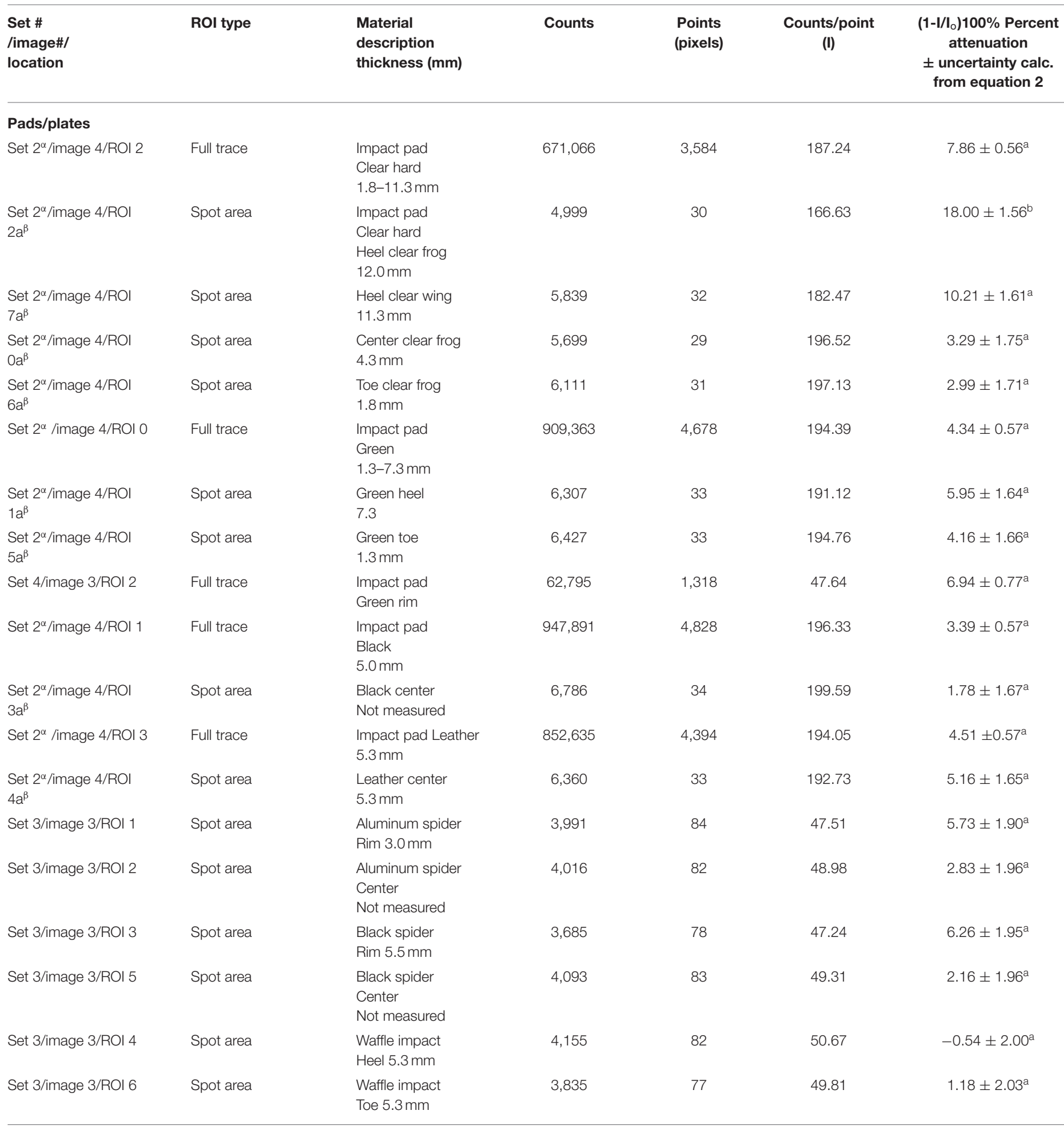

${ }^{\alpha}$ Percent Attenuation and regions of interest (RO/s)were calculated using Image 4 in Set 2 that was acquired for 12,000,000 counts to improve the observer's ability to correctly identify the margins of the pads.

${ }^{\beta}$ Where a second copy of an image was used for additional ROls, a suffix designation "a" was added to indicate those ROls.

a The attenuation caused by these pads was not statistically differently from one another.

${ }^{b}$ The attenuation caused by the clear impact pad at the heel over the frog was statistically different from the attenuation caused by the rest of the pads $(P<0.05)$.

Most shoes have an irregular surface with shaping, grooves, and other structural features. Measurements are only of the thickest part of the profile and will lead to overestimated attenuation if raw data from Table 2 is used. For these reasons one would not expect the predicted attenuations to exactly match the observed attenuations. 


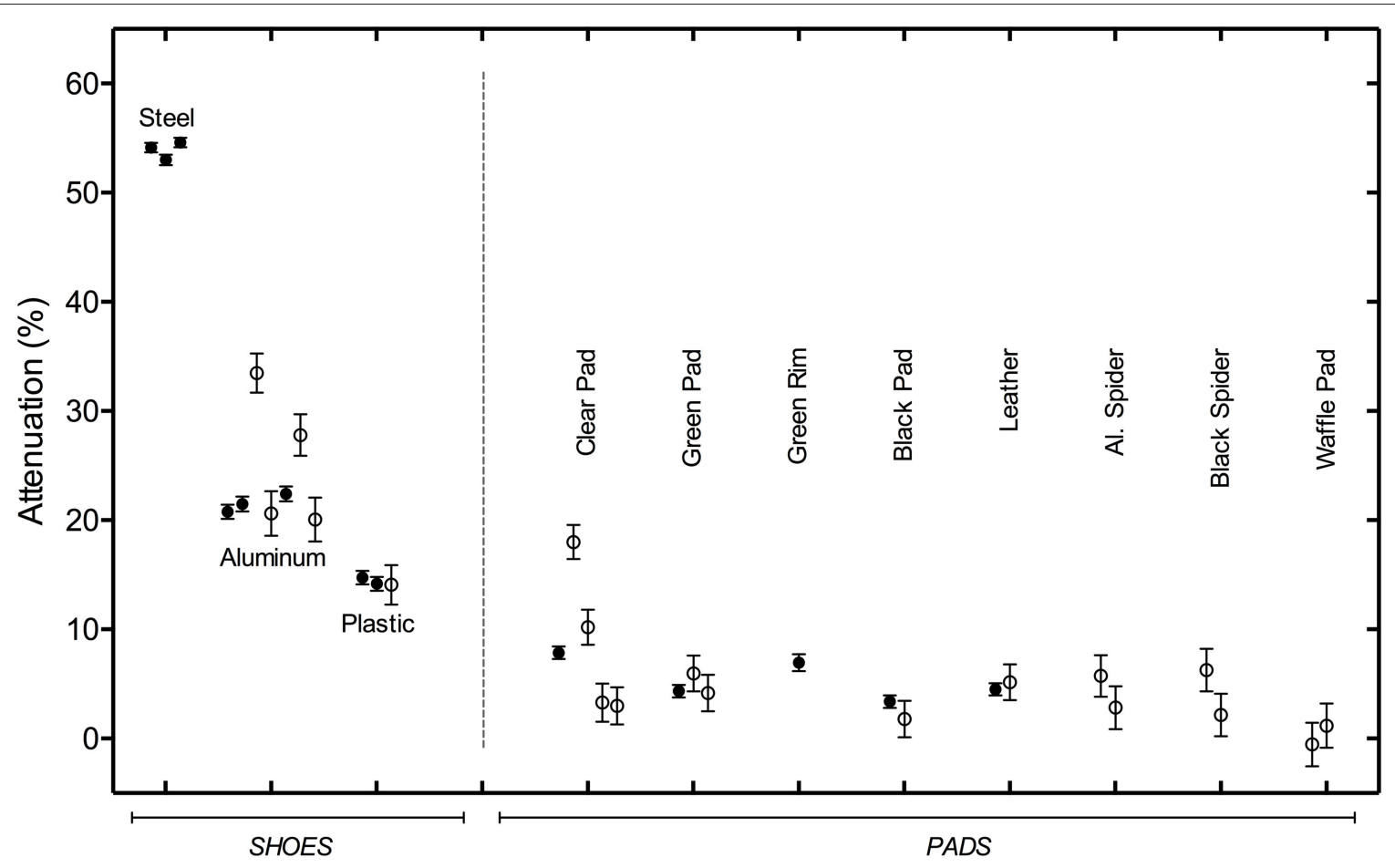

FIGURE 1 | Shoe and Pad Attenuation (with uncertainties). Attenuation for each shoe or pad imaged is shown as a circle for the mean attenuation, with error bars for the $95 \%$ confidence level. Filled circles indicate that the entire shoe or pad was traced, and open circles indicate ROI where a localized spot trace was used. Error bars on the spot traces are generally larger because of the smaller area included in the ROI. Two shoes were imaged twice, in different data sets. The difference shown for the two measurements of the attenuation of the small aluminum shoe and the plastic shoe shows how reproducibly the shoe outline could be traced.

TABLE 6 | Prediction of Attenuation for Different Materials and Thicknesses at $150 \mathrm{keV}$.

\begin{tabular}{|c|c|c|c|c|c|c|}
\hline Material & mass abs coeff(mac) & density(d) $\mathrm{g} / \mathrm{cm}^{3}$ & $\begin{array}{l}\text { representative } \\
\text { thickness(x) cm }\end{array}$ & Transmitted fraction $\mathrm{e}^{\left(-d^{\star} m a c^{*} x\right)}$ & predicted $\%$ attenuated & observed $\%$ attenuated \\
\hline \multicolumn{7}{|l|}{ Steel } \\
\hline & 0.184 & 8 & 0.50 & 0.479 & 52.1 & 54 \\
\hline & & & 0.75 & 0.332 & 66.8 & \\
\hline & & & 1.00 & 0.229 & 77.1 & \\
\hline \multicolumn{7}{|l|}{ Al } \\
\hline & 0.134 & 2.7 & 0.60 & 0.805 & 19.5 & 21 \\
\hline & & & 0.80 & 0.749 & 25.1 & \\
\hline & & & 1.00 & 0.696 & 30.4 & \\
\hline \multicolumn{7}{|l|}{ Polymer } \\
\hline & 0.134 & 1.2 & 1.00 & 0.851 & 14.9 & 14 \\
\hline & & & 1.50 & 0.786 & 21.4 & \\
\hline
\end{tabular}

To adequately appreciate the potential impact that the shoe and pad materials may have on nuclear scintigraphic images, it is important to understand the basic physics of gamma radiation and how images are generated.

The chemical and physical interaction of gamma-rays $(\gamma$ rays) with matter has been well-described in the literature (1618). Gamma radiation is absorbed in matter differently than absorption that occurs with charged particles. Its interaction is primarily with atomic electrons and the $\gamma$-ray can lose a large fraction, or all, of its energy in a single encounter. The rays are absorbed according to an exponential law, characterized by a half thickness and $\mu$ (linear absorption coefficient). The equation below provides the intensity $(I)$ of the rays after the initial intensity $\left(I_{0}\right)$ of a beam of $\gamma$-rays has passed through $x \mathrm{~cm}$ of absorbing matter.

$$
I=\operatorname{Io} e^{-\mu(\text { linear }) x}
$$


However, published values of material absorption coefficients $\mu$ (mass absorption) are expressed in units of $\left(\mathrm{cm}^{2} / \mathrm{g}\right)$, and to use those values to calculate expected attenuation values, you must also use the material density $\mathrm{D}\left(\mathrm{g} / \mathrm{cm}^{3}\right)$, to obtain the linear absorption coefficient $\mu$ as

$$
\mu=\mu(\text { linear })=D * \mu(\text { mass absorption })
$$

We can combine equations to get:

$$
\frac{I}{I o}=e^{-\mu(\text { mass absorption }) D x}
$$

where $I / I o$ is the fraction detected, $D$ is the density of the material in $\mathrm{g} / \mathrm{cm}^{3}, x$ is the thickness in $\mathrm{cm}$ and the mass absorption coefficient is in $\mathrm{cm}^{2} / \mathrm{g}$. Using density data for polyurethane, aluminum and steel, $\mu$ (mass absorption) and thicknesses measured from the materials in Table 2, we can predict attenuations of some materials in this study in Table 6. This validation of findings suggests that if a clinician knows the composition (density) and thickness of a given shoe or pad, a prediction of the percent attenuation can be made.

Compton scattering is the only source of extraneous radiation that could result from the shoe, because the photoelectric effect produces only electrons, not photons. However, in clinical practice typically two measures are used to prevent extraneous photons from being detected and interfering with the image generated from the source (i.e., the horse's foot). First, during image acquisition in vivo, the gamma camera is used with a lead collimator. The collimator narrows the beam of $\gamma$-rays, selecting only those that are aligned in a specific direction, namely, rays perpendicular to the camera sensor. Using the collimator in this study would have resulted in a non-uniform field and would have required much longer acquisition times (or a stronger source). Secondly, the camera software allows for "gating," whereby the photomultiplier tubes only detect $\gamma$-rays within a narrow range of energies (135-141 keV). This "gating" prevents photons of a lesser energy, such as those generated by Compton scattering, from being detected and interfering with the image and is used in this study and in the clinical setting. The image acquisition methodology used in this study also differs from what is done in a clinical setting. For this study, two modifications were used to create a uniform flood of gamma rays over the field: (1) the collimator was removed, and (2) the Tc source was placed several meters away from the sensor to effectively create a point source. A flood of the field was necessary in this study, as it is the only way to ensure uniformity of the gamma radiation hitting the detector and allows us to eliminate any potential variables affecting the data from a non-uniform field. The point source is several meters from the detector and therefore the photons hitting the detector are essentially at 90-degrees to the plane of the detector and the shoe is placed directly on the detector so no photons could hit the camera from other angles making the collimator unnecessary and will greatly reduce the count time. These two modifications do not mimic a clinical acquisition of data; however they were necessary to get an accurate calculation of the percent of attenuation and will give an accurate measure of the attenuating properties of the shoes and pads. In a clinical setting, the uptake of gamma radiation of interest is generated from bone, whereas the measurement of interest in this particular study is the interaction of the gamma radiation with the material and what is detected by the camera. Additionally, it was necessary to eliminate unknown variables such as movement, poor bone uptake and undiagnosed underlying pathology, from the current study to allow for comparative data for in vivo studies.

We propose that without removal of the shoe, the ability of the clinician to accurately identify the structure or structures associated with IRU on the solar view may be inhibited by attenuation of gamma radiation of clinically important structures and removing the shoe may improve the clinician's ability to make an accurate assessment of all the regions of interest. Additionally, we propose, when shod with a pad, there is further attenuation of the gamma radiation, which can further inhibit an accurate read of the solar image. Although plastic shoes caused significantly less attenuation than steel or aluminum shoes the attenuation is still considered statistically significant. Additionally, plastic shoes may not be a uniform thickness and often do not cover the entire sole, which may make clinical image interpretation in the presence of a plastic shoe difficult outside of just the percent attenuation caused by plastics of a uniform thickness. This concept is also illustrated by the measurements of clear plastic impact pad. The goal of this study was to attempt to quantify the amount of attenuation a given shoe or pad creates. Although attenuation is cumulative, it is not additive, because individual attenuations cannot simply be added to get the final attenuation for any given combination of materials. The progressive loss of transmission as it passes through multiple materials is multiplicative. An algebraic computation is required to represent the final amount of radiation attenuated. Accurate attenuation of individual materials is the focus of the current study. Since each material attenuates to some degree, there will be more attenuation when both a shoe and pad are present. This is can be considered a limitation in the current study, as pads will typically only be present in conjunction with shoes. Based on the results of this study, we believe the shoes should be pulled in any case where there are concerns that lameness originates from the foot.

Solar views in conjunction with the dorsal-palmar view have proven useful in assessment of the ungular (collateral) cartilages of the foot $(9,10)$. The aforementioned studies revealed an excellent agreement between radiographic and scintigraphic grades of ossification of the collateral cartilages and all fractured cartilages had a greater IRU. The agreement between IRU and lesion identification on MRI was significant for the palmar process and body of the distal phalanx (7). The region of insertion of the deep digital flexor tendon (DDFT) onto the coffin bone and collateral ligaments of the distal interphalangeal joint can also be evaluated on the solar view $(2,3,7)$. Current advances in Positron Emission Tomography (PET) with ${ }^{18} \mathrm{~F}-\mathrm{NaF}$ in equine diagnostic imaging shows promise in providing additional and valuable information in evaluation of structures in the equine distal $\operatorname{limb}(19,20)$. Although this modality utilizes similar nuclear medicine principles seen in 
standard equine bone scanning, the positrons are emitted at an energy of $511 \mathrm{keV}$, significantly higher than ${ }^{99 \mathrm{~m}} \mathrm{Tc}-\mathrm{MDP}$, and theoretically the attenuation would be reduced. Although the findings of this study may be applicable to this newer technique and could prevent accurate evaluation of images, PET is typically performed in conjunction with other imaging modalities (particularly computed tomography) which allow for more accurate localization of pathology and therefore require removal of the shoe.

Contrary to findings of the general musculoskeletal system, scintigraphy of the equine foot has relatively low sensitivity but is highly specific for injuries detected on MRI of the navicular bone, DDFT, and collateral ligaments of the distal interphalangeal joint $(1,2)$. Solar views have been used to detect abnormalities of the navicular bone by several authors $(1,2,5,14)$. There are two important considerations related to positioning of the navicular bone on the solar view that make this view extremely important (perhaps necessary) when evaluating potential pathology of the navicular bone. First, the width of the navicular bone in the transverse plane is greater than the height on the sagittal plane. Second, when the limb is positioned for a lateral view next to the gamma camera, the center of the navicular bone is at least half the width of the foot $(4.4-6.6 \mathrm{~cm})$ from the camera face and when positioned for the palmar views, the center of the navicular bone is much closer $(1-1.5 \mathrm{~cm})$ from the camera face (14). The distance from the collimator, overlying tissues and source dimensions affect the final image resolution $(14,21)$. This may be particularly important when evaluating horses with early or mild navicular abnormalities. In the authors experience, if a portion of the anatomy is attenuated, information about that part of the foot is lost and assessment of the image may be non-diagnostic. For example, steel shoes covering the heel and frog, will likely have a significant impact the ability to evaluate the navicular bone. This observation is quantified through this study which demonstrates that at least $50 \%$ of the radioactive emission is lost from the areas covered by the shoe and a smaller $(\leq 10 \%)$ amount is lost through the pads. Although the pads cause significantly less attenuation, it does not seem reasonable or technically feasible to remove only the shoes and leave the pads in place.

When the horse's foot is placed directly on the camera, any material placed between the radioactive bone and the detector will absorb some amount of radiation and therefore impact the image. In the authors experience, solar images where the shoe was present and then images were repeated with the shoes removed a significant loss of anatomic detail when the shoes were present was observed.

In a clinical setting, in the authors' experience, several interesting observations have been made. Structures emitting radiation from within the center of the shoe artificially appear more intense as the uptake from the solar margins of the coffin bone is reduced, particularly if the acquisition is based on obtaining a specific number of counts rather than acquiring for a specific amount of time. This effect is due to the attenuation from the shoes. Although this may seem beneficial in specific cases (i.e., suspected navicular disease), where the shoe is eliminating extraneous radiation from other structures within the foot, if information from the surrounding structures is lost the relative importance of the findings in the center of the foot may be unknown (i.e., there may be multiple problems in the foot of which one or more are not identified). Another clinical observation seen by the author, differentiation between focal IRU in the center of the foot (navicular bone) and more diffuse IRU toward the toe (associated with the coffin bone) is not as distinguishable with shoes as without the shoes. Possible reasons for this are the area of radioactive exposure from the foot is reduced in the images with shoes present, resulting in the structures in the center of the foot generating more counts relative to the rest of the foot. This effect may be particularly evident when considering the size of the horse's foot and relative size of the shoe. In horses with smaller feet, shod with shoes with wide bars, the width between the bars of the shoes becomes smaller and the effective area of the sole that is covered by the shoe increases and consequently decreases area of the sole that is unobstructed decreases. Although not measured in the current study, the width of the shoe and width of the bars of the shoe will likely play a significant role in attenuating the clinically important radiation coming from the foot when shoes are present. Another consideration affecting the clinical image is depending on the shoe, there is an additional $1-2 \mathrm{~cm}$ of distance between the radioactive source and the detector. This added distance reduces image resolution. Lastly, it is important to consider horses with poor perfusion of the distal limb, resulting in increased time to acquire a diagnostic image with adequate counts (22). This increased time for image acquisition may decrease the image resolution due to inherent motion of the patient.

We are well-aware that when foot lameness is a concern, shoes may play a vital role in the health of the horse's foot. Many horses require complex orthotic and therapeutic shoeing packages where cost may be a consideration relevant to the cost of the diagnostic imaging being performed. The imaging and removal of the shoes should be performed when the farrier is capable of putting the shoes back on in a reasonable amount of time. In the author's workplace, when the shoes are removed, the foot is carefully protected with tape immediately after the scan is performed. Foam or cotton sheet pads may also be used to provide added support after the scan is complete.

The findings of this study clearly demonstrate that there is unavoidable attenuation caused by shoes and associated footwear. This leads the authors to believe there is a significant advantage to imaging the horse's bare foot on the solar view and recommend removal of the shoe prior to bone scanning of the feet. As with other imaging techniques (radiography, MRI and CT) the necessity to remove the shoes due to technical difficulties and modality requirements are often understood by the referring veterinarian and owner. This study provides scientific evidence to the equine veterinary community and clients demonstrating the attenuation of gamma radiation caused by shoes and pads and provides room for in vivo studies demonstrating the need and advantage of removing the shoes/pads prior to the nuclear scintigraphic imaging process. 


\section{DATA AVAILABILITY STATEMENT}

The raw data supporting the conclusions of this article will be made available by the authors, without undue reservation, to any qualified researcher.

\section{AUTHOR CONTRIBUTIONS}

LW, MM, and NR contributed conception and design of the study. LW and MM contributed to interpretation and analysis of data. LW, MM, and BD wrote the first draft of the manuscript. LW, MM, BD, and ST participated in critical revision for intellectual content of the final manuscript. All authors read and approved the submitted version.

\section{REFERENCES}

1. Dyson S, Murray R. Verification of scintigraphy imaging for injury diagnosis in 264 horses with foot pain. Equine Vet J. (2007) 39:3505. doi: $10.2746 / 042516407 \times 185430$

2. Dyson SJ. Subjective and quantitative scintigraphic assessment of the equine foot and its relationship with foot pain. Equine Vet J. (2002) 2:16470. doi: 10.2746/042516402776767231

3. Martinelli M, Rantanen N. Relationship between nuclear scintigraphy and standing MRI in 30 horses with lameness of the foot. In: Proceedings of the Annual Convention of the American Association of Equine Practitioners. Seattle, WA (2005) p. 359-65.

4. Keegan KG, Wilson DA, Lattimer JC, Twardock AR, Ellersieck MR. Scintigraphic evaluation of $99 \mathrm{~m}$ Tc-methylene diphosphonate uptake in the navicular area of horses with lameness isolated to the foot by anesthesia of the palmar digital nerves. Am J Vet Res. (1996) 57:415-21.

5. Dyson S, Murray R, Schramme M, Blunden T. Current concepts of navicular disease. Equine Vet Educ. (2010) 23:2736. doi: 10.1111/j.2042-3292.2010.00172.x

6. Dyson S, Murray R. Collateral desmitis of the distal interphalangeal joint in 62 horses (January 2001-Dec2003). In: Proceedings of the Annual Convention of the American Association of Equine Practitioners. Denver, CO (2004) p. 248-256.

7. Quiney LE, Ireland JL, Dyson S. Evaluation of the diagnostic accuracy of skeletal scintigraphy for the causes of front foot pain determined by magnetic resonance imaging. Vet Radiol Ultrasound. (2018) 59:4908. doi: 10.1111/vru.12627

8. Keegan KG, Twardock AR, Losonsky JM, Baker GJ. Scintigraphic evaluation of fracture of the distal phalanx in horses:27 cases (1979-1988). J Am Vet Med Assoc. (1993) 202:1993-7.

9. Ruohoniemi M, Makela O, Eskonen T. Clinical significance of ossification of the cartilages of the front feet based on nuclear bone scintigraphy, radiography and lameness examination in 21 Finnhorses. Equine Vet J. (2004) 36:1438. doi: 10.2746/0425164044868729

10. Dyson SJ, Murray RC. Injuries associated with ossification of the cartilages of the foot. In: Proceedings of the Annual Convention of the American Association of Equine Practitioners. Baltimore, MD (2010) p. 152-65.

11. Nagy A, Dyson S, Murray R. Scintigraphic examination of the cartilages of the foot. Equine Vet J. (2007) 39:250-56. doi: 10.2746/042516407x171174

12. Ritmeester AM, Blevins WE, Ferguson DW, Adams SB. Digital perfusion, evaluated scintigraphically and hoof wall growth in horses with chronic laminitis treated with egg bar-heart bar shoeing and coronary grooving. Equine Vet J Suppl. (1998) 26:111-18.

13. Quiney LE, Ireland J, Dyson SJ. Evaluation of the diagnostic accuracy of skeletal scintigraphy in lame and poorly performing sport horses. Vet Radiol Ultrasound. (2018) 59:477-89. doi: 10.1111/vru.12626

\section{ACKNOWLEDGMENTS}

The authors would like to thank Robert Walker and Laurie Walker for multidisciplinary resources for physics of gamma radiation, project design, and statistical analysis. The authors would also like to thank Dr. Donna Shettko for writing assistance. The authors would also like to thank Mike Molnar for his technical assistance in utilizing the instrumentation.

\section{SUPPLEMENTARY MATERIAL}

The Supplementary Material for this article can be found online at: https://www.frontiersin.org/articles/10.3389/fvets. 2020.516718/full\#supplementary-material

14. Dyson S, Murray R. Use of concurrent scintigraphic and magnetic resonance imaging evaluation to improve understanding of the pathogenesis of injury of the podotrochlear apparatus. Equine Vet J. (2007) 39:36569. doi: 10.2746/042516407X185494

15. Dyson SJ, Pilsworth RC, Twardock AR, Martinelli MJ. Equine Scintigraphy. Newmarket: Equine Veterinary Journal Ltd. (2003). p. 68.

16. Harvey BG. Introduction to Nuclear Physics and Chemistry. Englewood Cliffs: Prentice Hall. (1969). p. 320-26.

17. Hubbell JH. Photon Cross Sections, Attenuation Coefficients and Energy Absorption Coefficients from $10 \mathrm{keV}$ to $100 \mathrm{GeV}$. Washington. (1969) Available online at: https://nvlpubs.nist.gov/nistpubs/Legacy/NSRDS/nbsnsrds29.pdf (accessed April 3, 2019).

18. Parks JE. The Compton Effect - Compton Scattering and Gamma Ray Spectroscopy. Knoxville. (2015) Available online at: http://www.phys.utk.edu/ labs/modphys/Compton\%20Scattering\%20Experiment.pdf (accessed April 3, 2019).

19. Spriet M, Espinosa P, Kyme AZ, Phillips KL, Katzman SA, Gluppo LD, et al. F-sodium fluoride positron emission tomography of the equine distal limb: exploratory study in three horses. Equine Vet J. (2018) 50:12532. doi: $10.1111 /$ evj.12719

20. Spriet M, Espinosa-Mur P, Cissell DD, Phillips KL, Arino-Estrada G, Beylin D, et al. F-sodium flouride positron emission tomography of the racing thoroughbred fetlock: validation and comparison with other imaging modalities in nine horses. Equine Vet J. (2019) 51:375-83. doi: 10.1111/evj.13019

21. Nagy A, Dyson S, Murray R. Radiographic, scintigraphic and magnetic resonance imaging findings in the palmar processes of the distal phalanx. Equine Vet J. (2008) 40:57-63. doi: 10.2746/042516407X22370

22. Dyson S, Lakhani K, Wood J. Factors influencing blood flow in the equine digit and their effect on uptake of $99 \mathrm{~m}$ technetium methylene diphosphonate into bone. Equine Vet J. (2001) 33:591-8. doi: 10.2746/042516401776563409

Conflict of Interest: LW, MM, NR, and BD were employed by the company California Equine Orthopedics and ST is employed by Blue Ridge Equine Clinic.

The authors declare that the research was conducted in the absence of any commercial or financial relationships that could be construed as a potential conflict of interest.

Copyright (c) 2020 Walker, Martinelli, Rantanen, Drumond and Trostle. This is an open-access article distributed under the terms of the Creative Commons Attribution License (CC BY). The use, distribution or reproduction in other forums is permitted, provided the original author(s) and the copyright owner(s) are credited and that the original publication in this journal is cited, in accordance with accepted academic practice. No use, distribution or reproduction is permitted which does not comply with these terms. 